\title{
Cancer-Related Fatigue After Esophageal Cancer Surgery: Impact of Postoperative Complications
}

\author{
Zhao Cheng, MD ${ }^{1}$, Asif Johar, MSc ${ }^{1}$, Magnus Nilsson, $\mathrm{MD}, \mathrm{PhD}^{2,3}$, and Pernilla Lagergren, $\mathrm{RN}, \mathrm{PhD}^{1,4}$ (i) \\ ${ }^{1}$ Surgical Care Science, Department of Molecular Medicine and Surgery, Karolinska Institutet, Karolinska University \\ Hospital, Stockholm, Sweden; ${ }^{2}$ Division of Surgery, Department of Clinical Science, Intervention and Technology \\ (CLINTEC), Karolinska Institutet, Stockholm, Sweden; ${ }^{3}$ Department of Upper Abdominal Diseases, Karolinska University \\ Hospital, Stockholm, Sweden; ${ }^{4}$ Department of Surgery and Cancer, Imperial College London, London, UK
}

\begin{abstract}
Background. The impact of postoperative complications on cancer-related fatigue is unknown. This nationwide prospective cohort study aimed to assess the trajectory of cancer-related fatigue and the influence of predefined postoperative complications on cancer-related fatigue up to 2 years after esophageal cancer surgery.
\end{abstract}

Methods. The patients in this study underwent esophagectomy between 2013 and 2019 in Sweden. The exposure was predefined postoperative complications. The outcome was cancer-related fatigue measured by the fatigue scale of the European Organization for Research and Treatment of Cancer Quality of Life Questionnaire Core 30 (EORTC QLQ-C30) and the EORTC QLQ-Fatigue 12 (QLQ-FA12) questionnaire. Linear mixed-effects models provided adjusted fatigue scores and mean score differences (MDs) with 95\% confidence intervals (CIs) between patients with and without predefined complications.

Results. The study enrolled 331 patients. The QLQ-C30 fatigue score increased with clinical relevance among patients with any complications (MD, 5.8; 95\% CI, 2.6-9.0) who had a higher Clavien-Dindo classification (grades 2 to 3a: MD, 7.3; 95\% CI, 3.1-11.5), a medical complication (MD, 6.9; 95\% CI, 3.0-10.7), or a pulmonary complication (MD, 6.9; 95\% CI, 2.1-11.6) for 1-1.5 years and remained stable until 2 years after esophagectomy.

(C) The Author(s) 2021

First Received: 6 July 2021

Accepted: 21 October 2021;

Published Online: 23 November 2021

P. Lagergren, RN, PhD

e-mail: pernilla.lagergren@ki.se
Similar patterns were found in the QLQ-FA12 fatigue and QLQ-FA12 physical and emotional subscales, but not in the cognitive subscales.

Conclusions. Complications in general and medical and pulmonary complications in particular might be associated with increased cancer-related fatigue after esophagectomy.

Cancer-related fatigue is a frequently reported distressing sense of tiredness related to both cancer and cancer treatment, including surgery-related fatigue, which cannot not be alleviated by rest or sleep. ${ }^{1,2}$ Cancer patients and survivors usually complain about incomplete role and activity involvement due to the lack of energy, and their quality of life is affected in all dimensions throughout their whole survivorship. ${ }^{3,4}$

Cancer-related fatigue is prevalent across different cancer survivors but may vary between cancer types. ${ }^{5-7}$ Besides, the understanding of cancer-related fatigue among specific cancer types, particularly cancer types with poor prognosis and survivorship, still is lacking and warranted.

In 2018, esophageal cancer ranked seventh in cancer incidence and sixth in cancer mortality globally. ${ }^{8}$ Esophagectomy remains the curatively intended treatment, followed by a high risk ( $>50 \%)$ of postoperative complications, ${ }^{9,10}$ and the 5-year survival is only about $30-50 \%$ after the surgery. ${ }^{11-13}$

Recent Swedish cohort studies found that postoperative complications had a negative impact on health-related quality of life after esophagectomy. ${ }^{14,15}$ However, the role of complications in cancer-related fatigue is only roughly reported, and fewer studies have examined specific conditions of complications in cancer-related fatigue. Such knowledge could help identify patient subgroups under 
increased burden of cancer-related fatigue. Detailed and reliable data about the development of cancer-related fatigue also could deepen the understanding of this disorder and facilitate the development of interventional strategies.

Therefore, this study aimed to measure cancer-related fatigue over time and to explore whether specific postoperative complications are followed by more severe cancerrelated fatigue among esophageal cancer survivors.

\section{METHODS}

\section{Study Design}

This study was based on an ongoing Swedish nationwide, prospective cohort entitled the Oesophageal Surgery on Cancer Patients-Adaptation and Recovery (OSCAR) study." The OSCAR includes esophageal cancer survivors in Sweden surgically treated since 1 January 2013 and onward. The project was approved by the Regional Ethical Review in Stockholm Board (diary no. 2013/844-31/1), and the informed consent forms were signed by all the participants.

\section{Data Source and Data Collection}

Patients were identified through collaboration with the pathology departments of all eight hospitals performing esophagectomies in Sweden, and the study enrolled the patients who survived 1 year after esophagectomy and were able to participate in the study.

Seven assessments were performed between 1 and 5 years after esophagectomy. The first assessment was performed 1 year after esophagectomy by a research nurse who visited the patients in their homes to guide them through the computer-based questionnaires for collecting data on patient-reported outcomes including cancer-related fatigue. Thereafter, at 1.5, 2, 2.5, 3 and 4 years postoperatively, patients were contacted by the project coordinator and responded to the written questionnaires sent by post. At the 5-year assessment, the research nurse visited the patients and performed the final interview in their homes. For the purpose of this study, all available data up to and including the 2-year follow-up period were used.

Clinical data from the time of surgery were collected by review of medical records according to a predefined protocol to ensure data consistency, including information on pathologic tumor stage, tumor histologic type, treatment, and postoperative complications. Comorbidity data were extracted from the Patient Registry. ${ }^{16}$ Education information was retrieved from the Longitudinal Integration Database for Health Insurance and Labor Market. Body weight data were collected from the medical records and follow-up measurements. Data linkages of the participants were enabled by the unique individual Swedish personal identity number, a 10-digit number assigned to each Swedish resident. ${ }^{17}$

\section{Exposure}

The study exposure was complications, defined as deviations from the normal postoperative course within 30 days after surgery. The included complications with definitions are descripted in Table 2.

\section{Outcome}

The primary outcome was cancer-related fatigue measured at $1,1.5$ and 2 years after esophagectomy. Two questionnaires developed by the European Organization for Research and Treatment of Cancer (EORTC) were used in this study: the fatigue scale of EORTC Quality of Life Questionnaire Core 30 (EORTC QLQ-C30) and an additional module, the EORTC QLQ-Fatigue 12 (EORTC QLQ-FA12). Both questionnaires are validated and sensitive to change. ${ }^{18,19}$

The QLQ-C30 is a 30-item questionnaire evaluating the quality of life of cancer patients, within which cancer-related fatigue is measured by a three-item scale. The QLQFA12 is a multidimensional instrument for the measurement of cancer-related fatigue with physical, cognitive, and emotional subscales in conjunction with the QLQ-C30. Fatigue scores from the questionnaires were transformed into scales of $0-100$. Missing data for each item were handled in line with the EORTC scoring manual. Higher scores indicate more cancer-related fatigue.

\section{Statistical Analysis}

Complications were analyzed in three ways: (1) occurrence of any complications (no or yes), (2) Clavien-Dindo classification $(0-1,2-3 a$, or $3 b-4),{ }^{20}$ and (3) four specific complication groups (no or yes): surgical complication, medical complication, pulmonary complication, and cardiac complication.

To account for the patient fatigue level before cancer diagnosis, ${ }^{3,5}$ the QLQ-C30 fatigue score from our Background Population study cohort, a random sample from the Swedish population, was used to calculate a proxy baseline score. Each patient was matched to 45 individuals, on the average, from the Background Population study cohort by age at surgery (5-year time window), sex, education level, and comorbidities. ${ }^{21}$ In addition, the proxy baseline score was calculated as the mean fatigue score of the matched individuals. 
Linear mixed-effects models were used to assess the fatigue scores up to 2 years after esophagectomy, with adjustment for the following confounders: proxy baseline QLQ-C30 fatigue score, age at surgery (continuous variable), sex (male or female), education level $(<9,9-12$ or $>12$ years of formal education), pathologic tumor stage (0-1, 2, or 3-4), neoadjuvant therapy (no or yes), Charlson Comorbidity Index $(0,1$, or $\geq 2)$, tumor histology (adenocarcinoma or squamous cell carcinoma), and weight change 1 year after the surgery (continuous variable). Additional analyses were conducted with further adjustment for the preoperative weight change (the difference between average weight as an adult and weight at operation).

The model included time as a categorical variable to allow for non-linear trajectories. Two-way interaction between exposure and time was included in the model to test the differences in fatigue trajectories between the patients with and without predefined complications. The fixed effects included all the covariates, time, and the interaction between complication and time. The intercept was estimated as a random effect to allow for variability across patients.

The results are presented as model-derived mean scores and mean score differences (MDs) with 95\% confidence intervals (CIs) of cancer-related fatigue. The MDs were estimated in two ways to facilitate interpretation: (1) MDs within predefined complication groups over time with the 1-year measurement as a reference and (2) MDs between predefined complication groups at each time point. On the scale of transformed score, MDs of 5-10 indicated small clinical relevance, MDs of 10-15 indicated medium clinical relevance, and MDs higher than 15 indicated large clinical relevance. ${ }^{22,23}$ The mixed-effects model allows for missing measurements within individuals. Thus all available data were used. ${ }^{24}$

A sensitivity analysis was performed, excluding observations from a patient who died within 2 months of the fatigue response to remove the impact of potential cancer recurrence on the outcome. An experienced biostatistician (A.J.) was responsible for the statistical analyses, and SAS 9.4 (SAS Institute, Cary, NC, USA) software was used for all analyses.

\section{RESULTS}

\section{Patients}

Between January 2013 and May 2019, 839 patients underwent esophagectomy for esophageal cancer in Sweden. Among these patients, 204 (24.3\%) died within 1 year and $113(13.5 \%)$ could not be reached, leaving 522 eligible patients for inclusion in the study. Of the eligible patients, $174(33.3 \%)$ were too sick or did not want to participate and $17(3.3 \%)$ were excluded due to unavailability of clinical data. Thus, 331 patients $(63.4 \%)$ were included in the 1-year measurement of the current study. At 1.5 years, 306 of these patients were alive, and 231 (75.5\%) completed the questionnaires. At 2 years, 259 were alive, and $182(70.3 \%)$ remained in the cohort.

Most of the covariates were distributed evenly between the patients with and without complications (Table 1). In the total cohort, $211(63.7 \%)$ of the patients had at least one postoperative complication. The five most common complications were pneumonia $(n=65,19.6 \%)$, atrial

TABLE 1 Demographics and clinical characteristics of the 331 patients who underwent esophagectomy for esophageal cancer in Sweden

\begin{tabular}{|c|c|c|}
\hline & \multicolumn{2}{|c|}{ Complication $n(\%)$} \\
\hline & No & Yes \\
\hline Total & $120(36.3)$ & $211(63.7)$ \\
\hline \multicolumn{3}{|l|}{ Age at operation } \\
\hline Mean & $66.5 \pm 8.0$ & $67.3 \pm 8.6$ \\
\hline \multicolumn{3}{|l|}{ Sex } \\
\hline Male & $14(11.7)$ & $20(9.5)$ \\
\hline Female & $106(88.3)$ & $191(90.5)$ \\
\hline \multicolumn{3}{|l|}{ Education level (years) } \\
\hline$<9$ & $26(21.7)$ & $49(23.2)$ \\
\hline $9-12$ & $51(42.5)$ & $83(39.3)$ \\
\hline$>12$ & $29(24.2)$ & $56(26.6)$ \\
\hline Missing & 14 (11.6) & $23(10.9)$ \\
\hline \multicolumn{3}{|l|}{ Weight loss (kg) } \\
\hline Mean & $8.8 \pm 7.8$ & $9.4 \pm 8.8$ \\
\hline Missing & $3(0.03)$ & $13(0.06)$ \\
\hline \multicolumn{3}{|l|}{ Pathologic tumor stage } \\
\hline $0-1$ & $42(35.0)$ & $69(32.7)$ \\
\hline 2 & $40(33.3)$ & $68(32.3)$ \\
\hline $3-4$ & $38(31.7)$ & $72(34.1)$ \\
\hline Missing & $0(0.0)$ & $2(0.9)$ \\
\hline \multicolumn{3}{|l|}{ Neoadjuvant therapy } \\
\hline No & $99(82.5)$ & $158(74.9)$ \\
\hline Yes & $19(15.8)$ & $49(23.2)$ \\
\hline Missing & $2(1.7)$ & $4(1.9)$ \\
\hline \multicolumn{3}{|l|}{ Tumor histology } \\
\hline Adenocarcinoma & $105(87.5)$ & $172(81.5)$ \\
\hline Squamous cell carcinoma & $15(12.5)$ & $39(18.5)$ \\
\hline \multicolumn{3}{|l|}{ Charlson comorbidity index } \\
\hline 0 & $60(50.0)$ & $84(39.8)$ \\
\hline 1 & 38 (31.7) & $71(33.7)$ \\
\hline$\geq 2$ & $22(18.3)$ & $56(26.5)$ \\
\hline
\end{tabular}


fibrillation ( $n=57,17.2 \%)$, anastomotic insufficiency $(n=$ $54,16.3 \%)$, respiratory insufficiency $(n=52,15.7 \%)$, and sepsis $(n=34,10.3 \%)$ (Table 2).

\section{Fatigue Scores Within Predefined Complication Groups Over Time Points}

Over time, the trajectory of QLQ-C30 fatigue showed a clinically relevant increase among the patients with complications (MD, 5.8; 95\% CI, 2.6-9.0), a higher ClavienDindo classification (grades 2-3a: MD, 7.3; 95\% CI, 3.1-11.5), a medical complication (MD, 6.9; 95\% CI, 3.0-10.7), or a pulmonary complication (MD, 6.9; $95 \% \mathrm{CI}$, 2.1-11.6) in 1-1.5 years, then remained stable until 2 years after esophagectomy. But the developments of QLQ-C30 fatigue were almost identical between the patients with and without surgical or cardiac complication (Fig. 1).

Similar to QLQ-C30 fatigue, the QLQ-FA12 fatigue deteriorated for the patients with complications (MD, 7.2; 95\% CI, 4.9-9.5), a higher Clavien-Dindo classification (grades 2-3a: MD, 8.0; 95\% CI, 5.0-11.0), a medical complication (MD, 8.2; 95\% CI, 5.5-10.9), or a pulmonary complication (MD, 9.7; 95\% CI, 6.4-13.1) in 1-1.5 years, then remained stable up to 2 years postoperatively. Besides, the QLQ-FA12 fatigue trajectory for the patients with and without pulmonary complications showed different developments over time $\left(P_{\text {interaction }}=0.023\right)$. Again, the patients with and without surgical or cardiac complications showed similar QLQ-FA12 fatigue trajectories (Fig. 2).

As for the QLQ-FA12 subscales, like the patients with patterns of QLQ-C30 and QLQ-FA12 fatigue, the patients with complications, a higher Clavien-Dindo grade, a medical complication, or a pulmonary complication reported a greater burden of physical and emotional fatigue in 1-1.5 years, and the burden remained until 2 years postoperatively. However, no clinically relevant trajectory differences were found for cognitive fatigue (Figs. A1A3).

\section{Fatigue Scores Between Predefined Complication Groups at Each Time Point}

Clinically relevant fatigue score differences between the patients with and without predefined complications were found 1.5 years postoperatively. The patients with at least one complication had a higher QLQ-FA12 fatigue score with clinical relevance (MD, 5.3; 95\% CI, 0.4-10.2) than those without complications 1.5 years postoperatively. However, for QLQ-C30 fatigue, the difference was clinically relevant but did not reach the level of statistical significance (MD, 5.6; 95\% CI, - 0.7 to 12.0). More QLQC30 fatigue was found among the patients with a Clavien-
Dindo classification grade higher than $3 b$ (MD, 8.0; 95\% CI, 0.1-15.8) compared with grades $0-1$ at 1.5 years. Besides, the QLQ-FA12 fatigue score increased for the patients with a medical complication (MD, 5.0; 95\% CI, 0.2-9.7), notably for those with a pulmonary complication (MD, 5.8; 95\% CI, 0.7-11.0), at the 1.5-year follow-up evaluation. No clinically significant MDs were found regarding surgical or cardiac complications at any time points (Table 3 ).

The MDs of the QLQ-FA12 physical, emotional, and cognitive fatigue subscales at each of the time points showed similar patterns. All the clinically relevant MDs were found 1.5 years postoperatively. The patients who had complications experienced greater physical fatigue (MD, 6.5 ; 95\% CI, 0.2-12.8). As for the Clavien-Dindo classification, the patients with grades higher than $3 \mathrm{~b}$ had more physical fatigue (MD, 8.0; 95\% CI, 0.2-15.7), and the patients with grades 2 to $3 \mathrm{a}$ showed greater emotional fatigue (MD, 8.1; 95\% CI, 1.4-14.8) and cognitive fatigue (MD, 5.7; 95\% CI, 0.7-10.8) in contrast to the patients with a grade lower than 1. Medical complication was associated with increased physical fatigue (MD, 6.5; 95\% CI, 0.5-12.5). Pulmonary disease was associated with all three subscales of fatigue, specifically physical (MD, 6.8; $95 \%$ CI, 0.2-13.4), emotional (MD, 7.4; 95\% CI, 1.1-13.8), and cognitive (MD, 6.1; 95\% CI, 1.3-11.0) fatigue (Table S1).

The analyses with further adjustment for preoperative weight change provided similar results (Tables S2 and S3; Figs. A4-A8). The results of the sensitivity analysis, excluding the observations from patients who died within 2 months after the response, were almost the same as those stated earlier (data not shown).

\section{DISCUSSION}

This study indicated a high level of cancer-related fatigue in esophageal cancer survivors after esophagectomy. Postoperative medical and pulmonary complications were associated with an increased level of cancer-related fatigue.

To our knowledge, this is the first prospective and longitudinal study to measure cancer-related fatigue among esophageal cancer survivors. The well-validated questionnaires, the nationwide and population-study based design, and the reliable data source counterbalanced the risk of information bias and ensured the generalizability of the results. The EORTC QLQ-C30 fatigue scale emphasizes the physical aspect of fatigue, whereas the EORCT QLQFA12 also covers the emotional and cognitive fatigue properties, ${ }^{25}$ but very few studies have used both questionnaires, hampering the comprehensive understanding of the measurements. 
TABLE 2 Postoperative complications within 30 days after esophagectomy for esophageal cancer for 331 patients

\begin{tabular}{llr}
\hline Complication & Definition & $n(\%)$ \\
\hline Complications & Occurrence of any complications & $120(36.3)$ \\
No & & $211(63.7)$ \\
Yes & - & $129(39.0)$ \\
Clavien-Dindo & & $116(35.0)$ \\
$\quad$ classification & & $85(25.7)$ \\
$0-1$ & & $1(0.3)$
\end{tabular}

Complication group ${ }^{\mathrm{a}}$

Medical complication

Surgical complication

Pulmonary complication

Cardiac complication

Complications type ${ }^{\mathrm{a}}$

Pneumonia

Atrial fibrillation

Anastomotic insufficiency

Respiratory insufficiency

Sepsis

Wound infection

Intrathoracic abscess or empyema

Recurrent laryngeal nerve paralysis

Pulmonary embolism

Thoracic ductus injury

Substitute necrosis

Intra-abdominal abscess

Myocardial infarction

Ileus

Renal failure

Postoperative bleeding

Other embolism

Strictures in anastomosis

Hepatic insufficiency

Gastric perforation

Wound dehiscence

Deep venous thrombosis

Cerebral infarction/stroke

Other complications ${ }^{\mathrm{b}}$
Sepsis, pneumonia, hepatic insufficiency, renal failure, deep venous thrombosis, pulmonary embolism, $146(44.1)$ other embolism, myocardial infarction, atrial fibrillation, cerebral infarction, or respiratory insufficiency

Postoperative bleeding, anastomotic insufficiency, substitute necrosis, thoracic ductus injury, intrathoracic abscess or empyema, intra-abdominal abscess, wound infection, wound dehiscence, ileus, gastric perforation, recurrent laryngeal nerve paralysis, or strictures in anastomosis

Respiratory insufficiency or pneumonia

Myocardial infarction or atrial fibrillation

Radiologically detected infiltrate with clinical symptoms such as fever, cough, or dyspnea

Newly electrocardiogram detected and treatment required

$57(17.2)$

Clinically significant or radiologically detected

Reintubation or mechanical ventilation needed

Causing clinical symptoms such as fever, chills, and proven bacteria in the blood

Causing clinical symptoms and requiring treatment

$\geq 3 * 3 \mathrm{~cm}$ radiologically or surgically detected abscess with clinical symptoms such as fever, pain or dyspnea

Laryngeal inspection ascertained

$22(6.6)$

Radiologically detected

Thoracic lymph leakage requiring drainage for more than 7 days or reoperation

$15(4.5)$

Clinically significant ischemia with ulceration or perforation

$11(3.3)$

$7(2.1)$

$\geq 3 * 3 \mathrm{~cm}$ radiologically or surgically detected abscess with clinical symptoms such as fever or pain

Radiologically detected ileus in need of surgery

$4(1.2)$

Dialysis needed

$>2000 \mathrm{ml}$ or requiring reoperation

Radiologically detected and requiring treatment

Endoscopic intervention required

Surgical intervention required

Clinically obvious wound rupture

Radiologically or clinically verified with treatment needs

Radiologically verified

$38(11.5)$

${ }^{a}$ Each patient could have more than 1 group or type of complications

${ }^{b}$ Most of the patients who had other complications also had the specific complication types listed above 

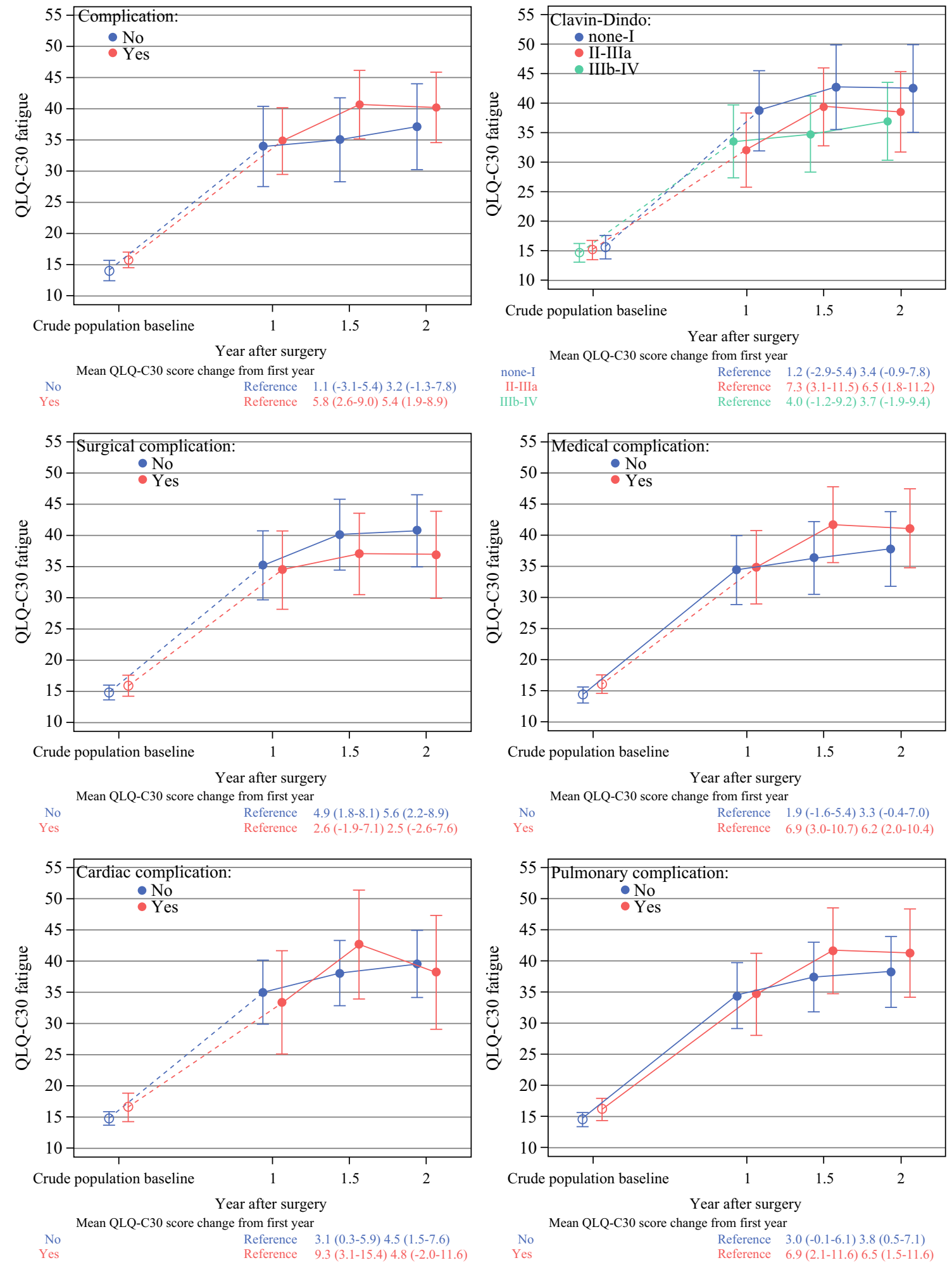

FIG. 1 QLQ-C30 fatigue trajectories and mean score differences with $95 \%$ confidence intervals between time points by predefined complications

This study provided complete results from the two commonly used questionnaires, thus filling the gap and enhancing the comparability with other studies. However, cognitive fatigue might have been underestimated in this study because severe cognitive fatigue could restrict 

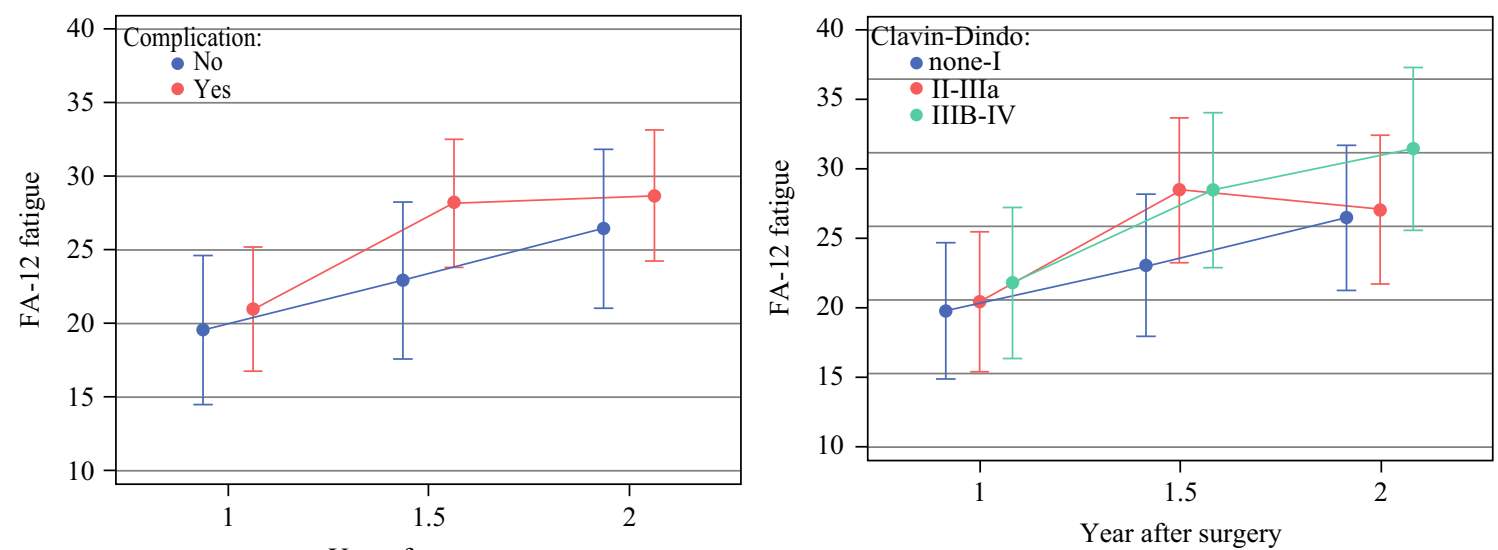

Year after surgery
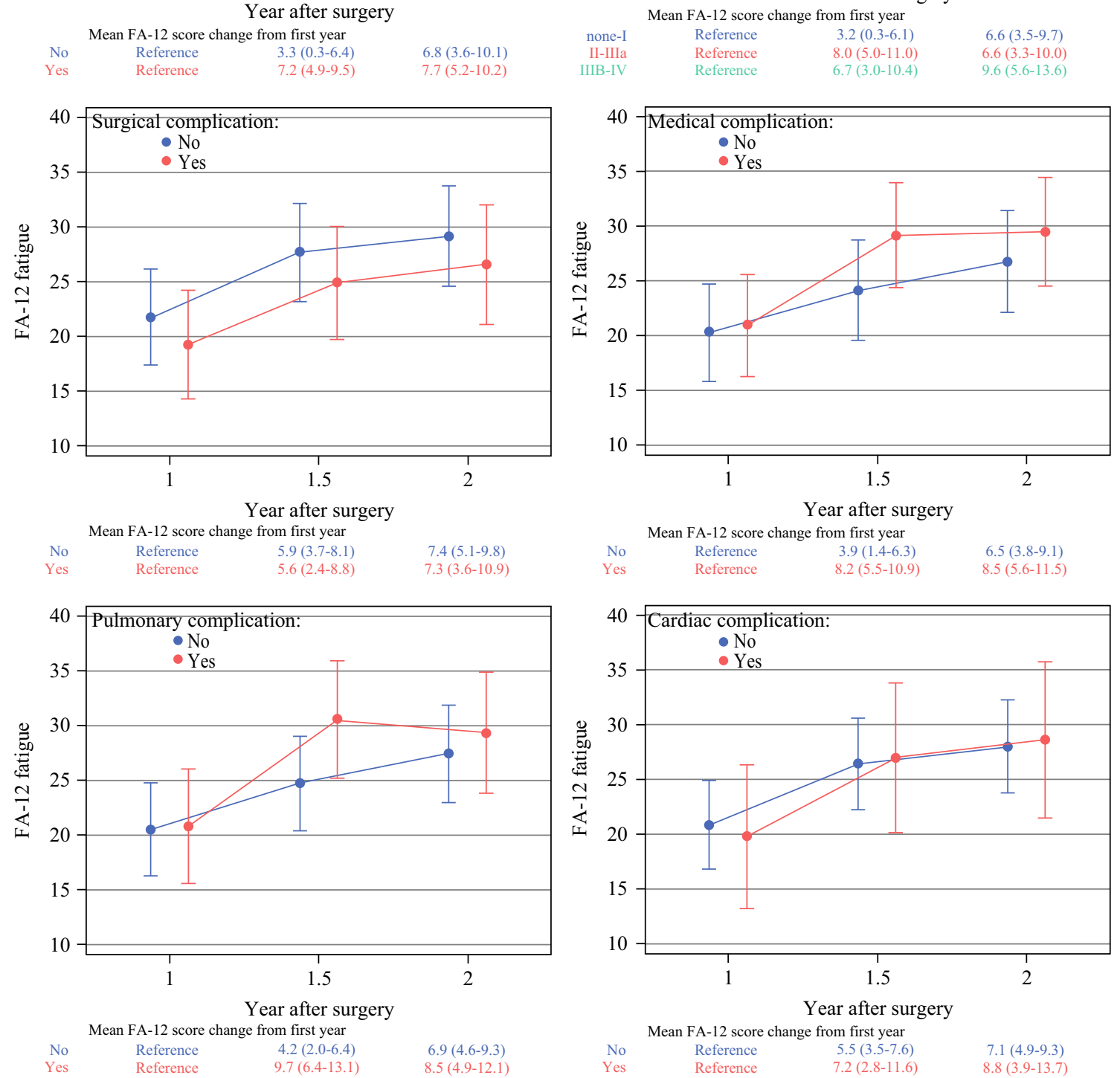

FIG. 2 QLQ-FA12 fatigue trajectories and mean score differences with $95 \%$ confidence intervals between time points by predefined complication.

patients' willingness or ability to participate in the study, indicating that the lack of association must be interpreted cautiously.
The lack of a baseline fatigue measurement was a weakness in this study, but a proxy baseline score from the matched reference cohort was calculated to mimic the 
TABLE 3 Adjusted mean score differences with $95 \%$ confidence intervals in QLQ-C30 and QLQ-FA12 fatigue scores between patients with and without predefined medical complications after esophagectomy at different time points ${ }^{\mathrm{a}}$

\begin{tabular}{|c|c|c|c|c|c|c|}
\hline & \multicolumn{3}{|l|}{ QLQ-C30 fatigue } & \multicolumn{3}{|l|}{ QLQ-FA12 fatigue } \\
\hline & 1 Year & 1.5 Years & 2 Years & 1 Year & 1.5 Years & 2 Years \\
\hline \multicolumn{7}{|c|}{ Complications } \\
\hline No & Reference & Reference & Reference & Reference & Reference & Reference \\
\hline Yes & $1.0(-4.9$ to 6.8$)$ & $5.6(-0.7$ to 12.0$)$ & $3.1(-3.6$ to 9.8$)$ & $1.4(-3.2$ to 6.0$)$ & $5.3(0.4-10.2)$ & $2.2(-2.9$ to 7.4$)$ \\
\hline \multicolumn{7}{|c|}{ Clavien-Dindo classification } \\
\hline $0-1$ & Reference & Reference & Reference & Reference & Reference & Reference \\
\hline $2-3 a$ & $-1.4(-7.9$ to 5.1$)$ & $4.7(-2.3$ to 11.6$)$ & $1.6(-5.8$ to 9.0$)$ & $0.6(-4.5$ to 5.7$)$ & $5.4(-0.0$ to 10.8$)$ & $0.6(-5.1$ to 6.3$)$ \\
\hline $3 b-4$ & $5.3(-2.0$ to 12.5$)$ & $8.0(0.1-15.8)$ & $5.6(-2.7$ to 13.8$)$ & $2.0(-3.7$ to 7.7$)$ & $5.4(-0.7$ to 11.5$)$ & $5.0(-1.4$ to 11.4$)$ \\
\hline \multicolumn{7}{|c|}{ Complication group } \\
\hline \multicolumn{7}{|c|}{ Surgical complication } \\
\hline No & Reference & Reference & Reference & Reference & Reference & Reference \\
\hline Yes & $-0.7(-6.8$ to 5.3$)$ & $-3.1(-9.7$ to 3.5$)$ & $-3.8(-10.9$ to 3.2$)$ & $-2.5(-7.3$ to 2.3$)$ & $-2.8(-7.9$ to 2.3$)$ & $-2.6(-8.1$ to 2.8$)$ \\
\hline \multicolumn{7}{|c|}{ Medical complication } \\
\hline No & Reference & Reference & Reference & Reference & Reference & Reference \\
\hline Yes & $0.4(-5.3$ to 6.1$)$ & $5.4(-0.7$ to 11.5$)$ & $3.3(-3.2$ to 9.8$)$ & $0.7(-3.8$ to 5.1$)$ & $5.0(0.2-9.7)$ & $2.8(-2.2$ to 7.8$)$ \\
\hline \multicolumn{7}{|c|}{ Pulmonary complication } \\
\hline No & Reference & Reference & Reference & Reference & Reference & Reference \\
\hline Yes & $0.3(-5.9$ to 6.5$)$ & $4.2(-2.5$ to 10.8$)$ & $3.0(-4.0$ to 10.0$)$ & $0.3(-4.6$ to 5.2$)$ & $5.8(0.7-11.0)$ & $1.9(-3.5$ to 7.3$)$ \\
\hline \multicolumn{7}{|c|}{ Cardiac complication } \\
\hline No & Reference & Reference & Reference & Reference & Reference & Reference \\
\hline Yes & $-1.6(-9.3$ to 6.1$)$ & $4.6(-3.6$ to 12.8$)$ & $-1.3(-10.1$ to 7.5$)$ & $-1.1(-7.2$ to 5.0$)$ & $0.6(-5.8$ to 7.0$)$ & $0.6(-6.2$ to 7.4$)$ \\
\hline
\end{tabular}

${ }^{a}$ Values in bold are both clinically relevant and statistically significant

fatigue level before cancer diagnosis and adjusted in the analysis to reduce concerns about the influence from host characteristics. Besides, an unmeasured or residual confounder, such as sarcopenia, was inevitable in this observational study. Moreover, some other postoperative complications, including diaphragmatic herniation and delayed gastric conduit emptying, were not available in the current cohort, which hampered the completeness of the assessment for the exposure. Dotted lines were used due to the lack of baseline measurement for the patients, and the fatigue trajectory between baseline and 1 year after esophagectomy could not be imputed. Another limitation was the potential selection bias caused by the patients who declined to participate due to serious illness and severe fatigue, but this could only dilute the associations and not reverse the results.

The esophageal cancer patients had higher fatigue levels after esophagectomy than the background population. One large longitudinal study also found higher fatigue scores among Hodgkin's lymphoma patients than among the German reference population, ${ }^{5}$ and studies regarding colorectal, breast, ovarian, and endometrial cancer reported a higher fatigue level than at the baseline before treatment. $^{26-28}$
However, the trajectories of fatigue vary among different cancer patients. Studies analyzing Hodgkin's lymphoma and breast cancer found that the fatigue scores increased dramatically during cancer therapy, then decreased to the pre-treatment level within 1 year and remained at a stable level. ${ }^{5,27}$ Nevertheless, an English cohort found that cancer-related fatigue also changed sharply during the first year after esophagectomy but kept at a higher level than the baseline before treatment. ${ }^{29}$

In the current study, data within 1 year after surgery were not available, but the fatigue score still increased between 1 and 1.5 years, and did not relieve until up to 2 years after the surgery. The reason might stem from the specific survival issues after esophagectomy. Esophageal cancer survivors experience psychiatric distress, eating difficulty, and physical symptoms of pain, cough, and reflux after the surgery, ${ }^{30}$ which could cause sleep disturbance and nutritional deficits, contributing to the poor survivorship with persisting cancer-related fatigue. ${ }^{3,4}$ This also could be the reason why the 1-year fatigue measurements are similar between patients with and those without predefined complications, considering that numerous strong factors exist during the initial postoperative period. 
As time passes after the surgery, some symptoms are relieved, the patients also gradually adapt to their new life, and the continuous effect of their complications show up.

In this study, the postoperative complications were grouped as surgical and medical complications. Surgical complications such as bleeding and anastomotic insufficiency are related to the surgical procedure, whereas medical complications usually are medical diseases such as pneumonia and myocardial infarction. Previous studies have found that postoperative complications, especially medical complications, were associated with long-lasting impaired health-related quality of life after esophagectomy. ${ }^{14,15}$

The current study provided further evidence regarding the effect of medical complications on cancer-related fatigue in detail. Patients with medical complications, specifically pulmonary complications, might already have chronic lung disease and worse performance status before the surgery. ${ }^{31,32}$ Such long-term comorbidities and complications that add disease burden ${ }^{5,26,27}$ and activate the inflammatory process and immune system reaction ${ }^{2,4}$ seem to increase the risk and severity of cancer-related fatigue. The effects of medical complications in the current study existed even after the adjustment for comorbidities. The lack of association between cardiac complications and cancer-related fatigue may be explained by the fact that the most common cardiac disorder, atrial fibrillation, was resolved in most cases at discharge and had no impact on long-term survival after esophagectomy. ${ }^{33,34}$ Previous Swedish studies have suggested that surgical complications healed in the long term, and that the influence on the QLQC30 fatigue score was diminished 5 years after esophagectomy. ${ }^{14,35}$ However, the current study found a limited effect of surgical complications on cancer-related fatigue 1 to 2 years after the surgery. A possible reason could have been the different calendar periods. The patients in the current cohort were surgically treated after 2013 and might have been relieved of surgical complications sooner due to the advanced surgical techniques and supportive care compared with the patients in the former studies conducted before 2005 .

The survival of esophageal cancer patients has been improving, and the way to promote the health-related quality of life after treatment has become an increasing interest for esophageal cancer patients together with the prolonged survival. Cancer-related fatigue is one of the most severe symptoms influencing the quality of life after esophagectomy, ${ }^{36}$ but no acknowledged treatment intervention has been discovered. This study provided evidence that reducing cancer-related fatigue could be achieved by the prevention of postoperative complications. Moreover, the results also emphasized the importance of considering individual effects of different complications. The risk of medical complications may be reduced by careful selection and optimization of patients, particularly pulmonary optimization. Minimally invasive surgery also is credited for the low incidence of pulmonary complications. ${ }^{37}$ As for patients with low pulmonary function before or after the surgery, rehabilitative intervention and long-term followup evaluation should be supported.

In summary, this prospective, population-based cohort study showed that medical and pulmonary complications might be associated with an increased level of cancer-related fatigue for esophageal cancer survivors. The trajectory of cancer-related fatigue increased in 1-1.5 years, then remained stable until 2 years after esophagectomy. These findings indicate the need for personalized and long-term follow-up evaluation for patients, accompanied by medical complications and the relevance of considering complications during the rehabilitation, with tailored support to counteract cancer-related fatigue after esophagectomy.

Supplementary Information The online version contains supplementary material available at https://doi.org/10.1245/s10434021-11049-z.

ACKNOWLEDGMENT This study was supported by the Swedish Cancer Society, the Stockholm County Council (ALF Project), the Cancer Research Funds of Radiumhemmet, the Swedish Research Council, and the China Scholarship Council.

FUNDING Open access funding provided by Karolinska Institute.

DISCLOSURE There are no conflicts of interest.

OPEN ACCESS This article is licensed under a Creative Commons Attribution 4.0 International License, which permits use, sharing, adaptation, distribution and reproduction in any medium or format, as long as you give appropriate credit to the original author(s) and the source, provide a link to the Creative Commons licence, and indicate if changes were made. The images or other third party material in this article are included in the article's Creative Commons licence, unless indicated otherwise in a credit line to the material. If material is not included in the article's Creative Commons licence and your intended use is not permitted by statutory regulation or exceeds the permitted use, you will need to obtain permission directly from the copyright holder. To view a copy of this licence, visit http://creativecommons. org/licenses/by/4.0/.

\section{REFERENCES}

1. Al Maqbali M, Al Sinani M, Al Naamani Z, Al Badi K, Tanash MI. Prevalence of fatigue in patients with cancer: a systematic review and meta-analysis. $J$ Pain Symptom Manage. 2021;61:167-89.

2. Fabi A, Bhargava R, Fatigoni S, et al. Cancer-related fatigue: ESMO Clinical Practice Guidelines for diagnosis and treatment. Ann Oncol. 2020;31:713-23.

3. Berger AM, Mooney K, Alvarez-Perez A, et al. Cancer-related fatigue. J Natl Compr Canc Netw. 2015;13:1012-39. 
4. Berger AM, Mitchell SA, Jacobsen PB, Pirl WF. Screening, evaluation, and management of cancer-related fatigue: ready for implementation to practice? CA Cancer J Clin. 2015;65:190-211.

5. Kreissl S, Mueller H, Goergen $\mathrm{H}$, et al. Cancer-related fatigue in patients with and survivors of Hodgkin's lymphoma: a longitudinal study of the German Hodgkin Study Group. Lancet Oncol. 2016;17:1453-62.

6. Jensen RE, Potosky AL, Moinpour CM, et al. United States population-based estimates of patient-reported outcomes measurement information system symptom and functional status reference values for individuals with cancer. J Clin Oncol. 2017;35:1913-20.

7. Schandl A, Johar A, Anandavadivelan P, Vikstrom K, Malberg K, Lagergren P. Patient-reported outcomes 1 year after oesophageal cancer surgery. Acta Oncol. 2020;59:613-9.

8. Bray F, Ferlay J, Soerjomataram I, Siegel RL, Torre LA, Jemal A. Global cancer statistics 2018: GLOBOCAN estimates of incidence and mortality worldwide for 36 cancers in 185 countries. CA Cancer J Clin. 2018;68:394-424.

9. Low DE, Kuppusamy MK, Alderson D, et al. Benchmarking complications associated with esophagectomy. Ann Surg. 2019;269:291-8.

10. Low DE, Alderson D, Cecconello I, et al. International consensus on standardization of data collection for complications associated with esophagectomy: Esophagectomy Complications Consensus Group (ECCG). Ann Surg. 2015;262:286-94.

11. Haverkamp L, Ruurda JP, van Leeuwen MS, Siersema PD, van Hillegersberg R. Systematic review of the surgical strategies of adenocarcinomas of the gastroesophageal junction. Surg Oncol. 2014;23:222-8.

12. Gottlieb-Vedi E, Kauppila JH, Mattsson F, et al. Long-term survival in esophageal cancer after minimally invasive esophagectomy compared to open esophagectomy. Ann Surg. 2021.

13. Shapiro J, van Lanschot JJB, Hulshof M, et al. Neoadjuvant chemoradiotherapy plus surgery versus surgery alone for oesophageal or junctional cancer (CROSS): long-term results of a randomised controlled trial. Lancet Oncol. 2015;16:1090-8.

14. Kauppila JH, Johar A, Lagergren P. Medical and surgical complications and health-related quality of life after esophageal cancer surgery. Ann Surg. 2020;271:502-8.

15. Kauppila JH, Johar A, Lagergren P. Postoperative complications and health-related quality of life 10 years after esophageal cancer surgery. Ann Surg. 2020;271:311-6.

16. Lagergren K, Derogar M. Validation of oesophageal cancer surgery data in the Swedish Patient Registry. Acta Oncol. 2012;51:65-8.

17. Ludvigsson JF, Otterblad-Olausson P, Pettersson BU, Ekbom A. The Swedish personal identity number: possibilities and pitfalls in healthcare and medical research. Eur $J$ Epidemiol. 2009;24:659-67.

18. Weis J, Tomaszewski KA, Hammerlid E, et al. International psychometric validation of an EORTC quality of life module measuring cancer-related fatigue (EORTC QLQ-FA12). J Natl Cancer Inst. 2017; 109.

19. Minton O, Stone P. A systematic review of the scales used for the measurement of cancer-related fatigue (CRF). Ann Oncol. 2009;20:17-25.

20. Dindo D, Demartines N, Clavien PA. Classification of surgical complications: a new proposal with evaluation in a cohort of 6336 patients and results of a survey. Ann Surg. 2004;240:205-13.

21. Derogar M, van der Schaaf M, Lagergren P. Reference values for the EORTC QLQ-C30 quality-of-life questionnaire in a random sample of the Swedish population. Acta Oncol. 2012;51:10-6.
22. Cocks K, King MT, Velikova G, et al. Evidence-based guidelines for interpreting change scores for the European Organisation for the Research and Treatment of Cancer Quality-of-Life Questionnaire Core 30. Eur J Cancer. 2012;48:1713-21.

23. Cocks K, King MT, Velikova G, Martyn St-James M, Fayers PM, Brown JM. Evidence-based guidelines for determination of sample size and interpretation of the European Organisation for the Research and Treatment of Cancer Quality of Life Questionnaire Core 30. J Clin Oncol. 2011;29:89-96.

24. Reidunsdatter RJ, Albrektsen G, Hjermstad MJ, Rannestad T, Oldervoll LM, Lundgren S. One-year course of fatigue after postoperative radiotherapy in Norwegian breast cancer patients: comparison to general population. Acta Oncol. 2013;52:239-48.

25. Kecke S, Ernst J, Einenkel J, Singer S, Hinz A. Psychometric properties of the fatigue questionnaire EORTC QLQ-FA12 in a sample of female cancer patients. J Pain Symptom Manage. 2017;54:922-8.

26. Poort $\mathrm{H}$, de Rooij $\mathrm{BH}$, Uno $\mathrm{H}$, et al. Patterns and predictors of cancer-related fatigue in ovarian and endometrial cancers: 1-year longitudinal study. Cancer. 2020;126:3526-33.

27. Person H, Guillemin F, Conroy T, Velten M, Rotonda C. Factors of the evolution of fatigue dimensions in patients with breast cancer during the 2 years after surgery. Int $J$ Cancer. 2020;146:1827-35.

28. Vardy JL, Dhillon HM, Pond GR, et al. Fatigue in people with localized colorectal cancer who do and do not receive chemotherapy: a longitudinal prospective study. Ann Oncol. 2016;27:1761-7.

29. Lagergren P, Avery KN, Hughes R, et al. Health-related quality of life among patients cured by surgery for esophageal cancer. Cancer. 2007;110:686-93.

30. Jacobs M, Macefield RC, Elbers RG, et al. Meta-analysis shows clinically relevant and long-lasting deterioration in health-related quality of life after esophageal cancer surgery. Qual Life Res. 2014;23:1155-76.

31. Grotenhuis BA, Wijnhoven BP, Grune F, van Bommel J, Tilanus HW, van Lanschot JJ. Preoperative risk assessment and prevention of complications in patients with esophageal cancer. J Surg Oncol. 2010;101:270-8.

32. Charalampakis N, Xiao L, Lin Q, et al. Co-morbidities rather than age impact outcomes in patients receiving preoperative therapy for gastroesophageal adenocarcinoma. Ann Surg Oncol. 2017;24:2291-301.

33. Mc Cormack O, Zaborowski A, King S, et al. New-onset atrial fibrillation post-surgery for esophageal and junctional cancer: incidence, management, and impact on short- and long-term outcomes. Ann Surg. 2014;260:772-8.

34. Chevallay M, Jung M, Chon SH, Takeda FR, Akiyama J, Monig S. Esophageal cancer surgery: review of complications and their management. Ann N Y Acad Sci. 2020;1482:146-62.

35. Rutegard M, Lagergren J, Rouvelas I, Lindblad M, Blazeby JM, Lagergren P. Population-based study of surgical factors in relation to health-related quality of life after oesophageal cancer resection. Br J Surg. 2008;95:592-601.

36. Schandl A, Johar A, Anandavadivelan P, Vikstrom K, Malberg K, Lagergren P. Patient-reported outcomes 1 year after oesophageal cancer surgery. Acta Oncol. 2020;59:613-9.

37. Biere SS, van Berge Henegouwen MI, Maas KW, et al. Minimally invasive versus open oesophagectomy for patients with oesophageal cancer: a multicentre, open-label, randomised controlled trial. Lancet. 2012;379:1887-92.

Publisher's Note Springer Nature remains neutral with regard to jurisdictional claims in published maps and institutional affiliations. 